\title{
Stress levels in high-school students after a semester of home-schooling
}

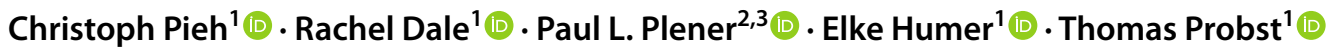

Received: 22 May 2021 / Accepted: 11 June 2021 / Published online: 16 June 2021

c) Springer-Verlag GmbH Germany, part of Springer Nature 2021

\begin{abstract}
There has been an increase in stress in adolescents since the beginning of the COVID-19 pandemic. Social distancing and home-schooling are just two of many stress factors for this age group. The aim of this study was to assess stress in high-school students after a semester of home-schooling. A cross-sectional online survey (February 3rd to 28th 2021) was performed, measuring stress with the Perceived Stress Scale (PSS-10) in Austria. In total, $N=2884$ students (age: $M=16.47$ (SD = 1.44); $70.4 \%$ females) completed the survey. Mean PSS-10 score was $M=23.50$ ( $\mathrm{SD}=7.47$ ) [females: $M=24.69$ ( $\mathrm{SD}=6.80)$; males: $M=20.11$ (SD=7.93); $p<0.001$ ]. 11.0\% reported low stress (females: 7.2\%; males 20.9\%), 52.5\% moderate stress (females: 51.5\%; males: 57.3\%), and 36.5\% high stress (females: $41.3 \%$; males 21.8\%); $p<0.001$. Around one-third of high-school students suffer from high stress level, females almost twice as often as males. Psychological support should particularly focus on stress and possibilities to reduce it.
\end{abstract}

\section{Introduction}

In light of recent publications related to the effects of the COVID-19 pandemic [1-3], we were interested in assessing perceived stress levels in adolescents after a semester of home-schooling and almost 1 year of social distancing in Austria.

The coronavirus (COVID-19) pandemic has been determining our everyday lives for more than a year. As in many other countries, Austria imposed lockdown measures, social distancing and home-schooling. All schoolchildren aged 14 or higher received almost exclusively home-schooling between October 2020 and February 2021.

A recent Australian study showed that the COVID-19 pandemic negatively affects mental health in adolescents, with $48 \%$ of $12-18$-year-old adolescents reporting psychological distress [1]. High stress levels in children and adolescents are reported in different countries [4-6]. An Italian

Christoph Pieh

Christoph.Pieh@donau-uni.ac.at

1 Department for Psychotherapy and Biopsychosocial Health, Danube University Krems, Krems, Austria

2 Department of Child and Adolescent Psychiatry, Medical University of Vienna, Vienna, Austria

3 Department of Child and Adolescent Psychiatry and Psychotherapy, University of Ulm, Ulm, Germany study from April 2020 found moderate or high stress levels in 29\% of 14-20-year-old adolescents [6]. An Indian study from April 2020 found severe stress measured with the PSS10 in $4.7 \%$ of adolescents and young adults [5]. Data for these studies were collected mainly at an earlier stage of the pandemic.

\section{Methods}

A cross-sectional study was conducted online using Research Electronic Data Capture (REDCap) from February 3rd to February 28th 2021 in 14- to 20-year-old adolescents. All Austrian schools were informed by the Austrian Federal Ministry of Education, Science and Research. All participants gave electronic informed consent before participation. The ethics committee of the Danube-University Krems (protocol code EK GZ 41/2018-2021) approved the study.

Stress was measured with the total scale of the Perceived Stress Scale (PSS-10). The PSS-10 is a validated and widely used 10-item questionnaire to self-rate stress [7] for the last month from 0 to 4 (maximum score 40; low stress: 0-13; moderate stress: 14-26; high stress 27-40). The German version is validated for adolescents aged 14 years or older [8]. Cronbach's alpha was $\alpha=0.87$ in the current sample. All data were analyzed using the IBM SPSS Statistics software version 26. Univariate ANOVAs and Chi-squared tests were 
computed to analyze differences in stress according to gender. The significance level was set at 0.05 (two-tailed).

\section{Results}

A total of $N=2884$ adolescents completed this study (70.4\% female, $27.9 \%$ male, $1.8 \%$ diverse; $16.1 \%$ with migration background). Participants were on average $M=16.47$ $(\mathrm{SD}=1.44)$ years old and representative according to regions for Austria.

Mean score for stress levels on the PSS-10 total scale was $M=23.50(\mathrm{SD}=7.47$ (females: $M=24.69(\mathrm{SD}=6.80)$; males: $M=20.11$ ( $\mathrm{SD}=7.93)$; diverse: $M=29.39$ $\left.(\mathrm{SD}=7.24) ; F(2 ; 2881)=136.12 ; p<0.001 ; \eta^{2}=0.086\right)$. In total, $36.5 \%$ scored above the cut-off score for high stress levels (PSS-10 mean score from 27 to 40; females: 41.3\%; males $21.8 \%$; diverse: $76.5 \%$ ), $52.5 \%$ moderate stress (PSS10: 14-26; females: 51.5\%; males: 57.3\%; diverse: $17.6 \%$ ), and $11.0 \%$ reported low stress (PSS-10: $0-13$, females: 7.2\%; males $20.9 \%$; diverse: $5.9 \%$ ), with a significant effect of gender on stress category $\left(\chi^{2}(4)=198.3, p<0.001\right)$.

\section{Discussion}

More than one-third of high-school students report a high level of perceived stress.

Taking into account those who have at least moderate stress, the prevalence rises to $89 \%$. Compared with studies at the beginning of the pandemic [4-6], stress is significantly more prevalent in adolescents as the pandemic continues. High stress levels are almost twice as common in females than in males. These results are in line with previous studies. For example, Nocentini et al. found stress in females 2.5 more prevalent than in males [6].

Several representative Austrian studies in adult samples consistently found the highest stress load in the late adolescents' age group from 18 to 24 years old [9-11]. However, the prevalence of highly stressed people increased significantly during the pandemic. While in April 2020, 4.2\% of the 18-24 years old scored above the cut-off for high stress level in Austria [9], it was $21.6 \%$ in the same age group around the turn of the year 2020/21 [11]. Even if these age groups, only slightly overlap with the current sample, a similar trend would also be possible for 14- to 20-year-olds.

The COVID-19 pandemic and the associated lockdown measures have multiple consequences on the lives of adolescents, such as home confinement, home-schooling, disruption of social contacts, worry for their families, worry for the future job situation as well as chronic and acute stress, among others. However, due to the study design, it is not possible to draw causal conclusions as to whether the increased stress level is due to the pandemic or the confinement measures such as lockdowns (e.g., separation from friends, school disruption, etc.), or even if there is a completely different, yet unknown cause.

This study has several further limitations, such as the cross-sectional design, the exclusive use of a self-rating instrument, or the possible self-selection bias due to the online setting. Moreover, the sample is representative for the region, but not for the gender ratio in Austria.

Overall, this study shows a high stress level in highschool students after a semester of home-schooling and almost a year of social distancing. Compared with data from the beginning of the pandemic, there is a significant increase, especially in the highly stressed group. While it is likely that the pandemic and lockdowns contribute to this development, this cannot be concluded based on the study design. Regardless, several attempts should be made to reduce this high level of stress in high-school students. It is crucial to carefully balance the potential benefits of reduced COVID-19 transmission with detrimental effects of homeschooling before further school closures are implemented during possible further waves of the COVID-19 pandemic.

Authors' contributions Conceptualization: CP, PLP, TP, EH. Data curation: EH. Statistical analysis: EH, RD. Investigation: CP, PLP, EH, TP. Methodology: CP, EH, PLP, RD. Project administration: CP, EH. Writing—original draft: CP, RD, TP. Writing—review and editing: EH, PLP.

Funding None.

Availability of data and materials The raw data supporting the conclusion of this article will be made available by the authors upon reasonable request.

\section{Declarations}

Conflict of interest None.

Ethical approval The principles outlined in the Declaration of Helsinki were followed and the ethics committee of the Danube-University Krems as well as the data protection officer of the Danube-University Krems approved the study (EK GZ 41/2018-2021).

Consent to participate All participating adolescents had to agree to the data protection declaration to start the survey (electronic informed consent).

Consent for publication Participants gave informed consent for publication.

\section{References}

1. Li SH, Beames JR, Newby JM, Maston K, Christensen H, Werner-Seidler A (2021) The impact of COVID-19 on the lives and 
mental health of Australian adolescents. Eur Child Adolesc Psychiatry 1-13. https://doi.org/10.1007/s00787-021-01790-x

2. Cost KT, Crosbie J, Anagnostou E et al. (2021) Mostly worse, occasionally better: impact of COVID-19 pandemic on the mental health of Canadian children and adolescents. Eur Child Adolesc Psychiatry 1-14. https://doi.org/10.1007/s00787-021-01744-3

3. Larsen L, Helland MS, Holt T (2021) The impact of school closure and social isolation on children in vulnerable families during COVID-19: a focus on children's reactions. Eur Child Adolesc Psychiatry 1-11. https://doi.org/10.1007/s00787-021-01758-x

4. Flaudias V, Iceta S, Zerhouni O et al (2020) COVID-19 pandemic lockdown and problematic eating behaviors in a student population. J Behav Addict 9(3):826-835. https://doi.org/10.1556/2006. 2020.00053

5. Agarwal N, Harikar MM, Shukla R, Bajpai A (2021) Factors determining psychological stress among Indian adolescents and young adults during the COVID-19 outbreak. Indian J Soc Psychiatry $37: 82-87$

6. Nocentini A, Palladino BE, Menesini E (2021) Adolescents' stress reactions in response to COVID-19 pandemic at the peak of the outbreak in Italy. Clin Psychol Sci 9(3):507-514. https://doi.org/ $10.1177 / 2167702621995761$
7. Cohen S, Kamarck T, Mermelstein R (1983) A global measure of perceived stress. J Health Soc Behav 24:385-396. https://doi.org/ $10.2307 / 2136404$

8. Klein EM, Brähler E, Dreier M et al (2016) The German version of the perceived stress scale-psychometric characteristics in a representative German community sample. BMC Psychiatry 16:159. https://doi.org/10.1186/s12888-016-0875-9

9. Pieh C, Budimir S, Probst T (2020) The effect of age, gender, income, work, and physical activity on mental health during coronavirus disease (COVID-19) lockdown in Austria. J Psychosom Res 136:110186. https://doi.org/10.1016/j.jpsychores.2020. 110186

10. Pieh C, Probst T, Budimir S, Humer E (2021) Diminished wellbeing persists beyond the end of the COVID-19 lockdown. Gen Hosp Psychiatr 70:137-138. https://doi.org/10.1016/j.genhosppsy ch.2021.01.004

11. Dale R, Budimir S, Probst T, Stippl P, Pieh C (2021) Mental health during the COVID-19 lockdown over the christmas period in Austria and the effects of sociodemographic and lifestyle factors. Int J Environ Res Public Health 18(7):3679. https://doi.org/ 10.3390/ijerph18073679 